\title{
Minimally Invasive Approach to Restore Anterior Spaces with Porcelain Laminate Veneers
}

\author{
Prasad Nanasaheb Mhaske, Sabita M Ram, Naisargi Shah, Jyoti B Nadgere
}

\begin{abstract}
Restoring anterior teeth is a big challenge for dentists today because of the esthetic concerns and also the patient's expectation for a natural looking smile. An unpleasant smile traumatizes the patient psychologically as it decreases their confidence and is a cause for social embarrassment. This case describes a patient who reported with a complaint of open spaces in maxillary anterior teeth, desiring enhancement of esthetics and was treated successfully with porcelain laminate veneers. Very minimal tooth preparation was carried out on the labial aspect of the maxillary incisors and canines, keeping in mind the minimally invasive approach. Veneers were fabricated using lithium disilicate ceramics and were bonded to the tooth surface using adhesive resin cement.
\end{abstract}

Keywords: Minimally invasive, Porcelain laminate veneers, Anterior spaces.

How to cite this article: Mhaske PN, Ram SM, Shah N, Nadgere JB. Minimally Invasive Approach to Restore Anterior Spaces with Porcelain Laminate Veneers. J Contemp Dent 2013;3(2):108-111.

Source of support: Nil

Conflict of interest: None declared

\section{INTRODUCTION}

Management of anterior teeth to enhance esthetics is one of the most difficult tasks encountered by a dentist. The patients may have discolored, fractured, attrided, malformed or malaligned teeth. ${ }^{1}$ They may also have a discrepancy with the tooth and jaw size. This may be due to genetic inheritance which is usually a combination of a large jaw from one parent and small teeth from other. Some of these discrepancies may be corrected with orthodontic alignment, but many a times, it is not possible to close the spaces in patients with smaller size teeth and larger jaws.

Restoring diastema in anterior teeth with laminate veneers is the most conservative and esthetic approach for management of anterior spacing. Veneers may be direct composite laminates or indirect porcelain laminates. However, the porcelain laminates have a greater advantage of esthetics over the composite laminates and give a lifelike appearance to the restoration. They have good survival rate if the case is correctly selected. The most important factor is the adhesion between the laminates and the underlying tooth surface. Therefore, the amount of enamel present plays an important role in the success of porcelain laminate veneers. ${ }^{2}$ To achieve predictable results, it is very important for the clinician to understand the philosophy of esthetic dentistry, the indications and contraindications, selection of laminate material, knowledge of tooth preparation and luting of laminates.

\section{CASE REPORT}

Here is a case report of a 52-year-old female patient who reported to the Department of Prosthodontics of the MGM. Dental College and Hospital, Navi Mumbai, with the chief complaint of an unpleasant smile. She desired enhancement in esthetics with closure of spaces between her upper front teeth (Fig. 1).

Patient was a pleasant looking lady until she smiled. The spacing between her teeth did not compliment her facial features. The teeth appeared smaller in size with large spacing between her upper central incisors. Intraoral examination revealed large midline diastema and spacing between central and lateral incisors, with the left lateral incisor placed more labially (Fig. 2). Presence of implant supported prostheses with 16, 46 and 47 and a tooth supported fixed prostheses with 35, 36 and 37.



Fig. 1: Pretreatment extraoral photograph



Fig. 2: Pretreatment intraoral view 


\section{Treatment Plan}

Orthodontic opinion was taken in which they opined that there was a discrepancy between the jaw size and size of the teeth and orthodontic treatment would have been a long time process to which the patient did not agree. Patient was given the option of porcelain laminate veneers since it was a minimally invasive treatment, less time consuming with immediate results. Upper and lower diagnostic impressions were made in irreversible hydrocolloid impression material and poured with type III dental stone. A diagnostic wax up was done over maxillary anterior teeth to get the proper size, form and proportion of the teeth (Fig. 3). This was presented to the patient, and approval was taken. The finalization of the laminates was done at the time of the intraoral composite mock up.

A silicone (Express ${ }^{\mathrm{TM}}$ XT Putty Soft, 3M ESPE, USA) index was made over the waxed up cast, one for temporization and another as a guide for tooth preparation. Shade selection was done prior to the tooth preparation and A3 was selected using shade guide (Vitapan Classical, Vita Zahnfabrik, Germany). In the silicone index, composite resin was applied in the region where laminates were planned and this was placed over the unprepared teeth to get the intraoral composite mock up. These were the pre-evaluated temporaries (Fig. 4). They were evaluated by both the dentist

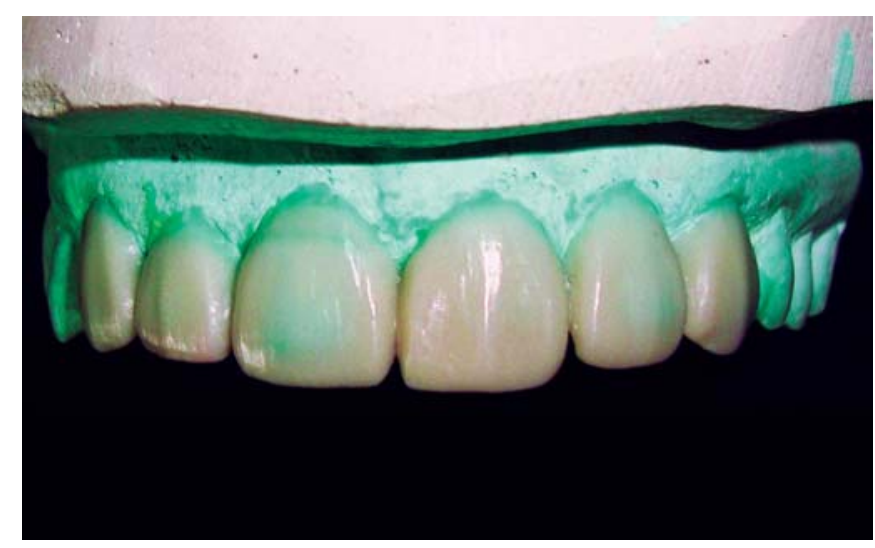

Fig. 3: Diagnostic wax up on cast

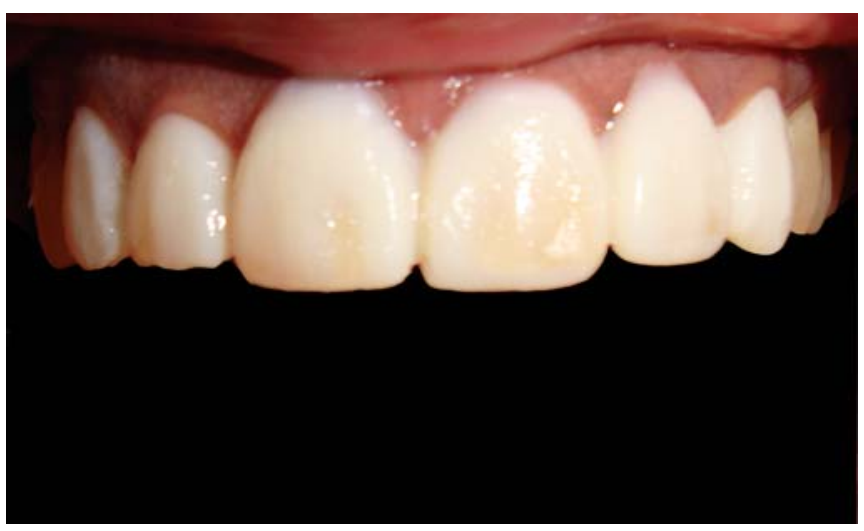

Fig. 4: Esthetic pre-evaluated temporaries and the patient and the final approval for the shape and size was taken from the patient.

As minimum preparation was required, the pre-evaluated temporaries were removed and minimum preparation on the labial surface of $0.5 \mathrm{~mm}$ was carried out with a definitive finishing line at the cervical margin with a round ended tapered fissure bur. Labial preparation was verified using silicone guide (Figs 5A and B). Preparation was done in such a way that reduction was done more on the distal aspect and minimal on the mesial aspect in order to close the mesial diastema. Equigingival finish line was placed on the labial aspect, no definitive finish line was placed on mesial and the distal side as diastema required to be closed. Incisal butt joint was given with minimal incisal preparation. The preparation was finished and gingival retraction was carried out using atraumatic method of gingival retraction (Traxodent, Premier restorative products) as shown (Fig. 6A). This was done to accurately record the gingival finishing line. After 10 minutes, the material was washed off the teeth surfaces and dried.

A stock metal tray was selected and coated with tray adhesive (Tray fix, Dentsply, Germany). Vinyl polysiloxane (Express ${ }^{\mathrm{TM}}$ XT Putty Soft and light body, 3M ESPE, USA) impression material was used to make a two-step impression to get the details of the preparation (Fig. 6B). Impression



Fig. 5A: Tooth preparation

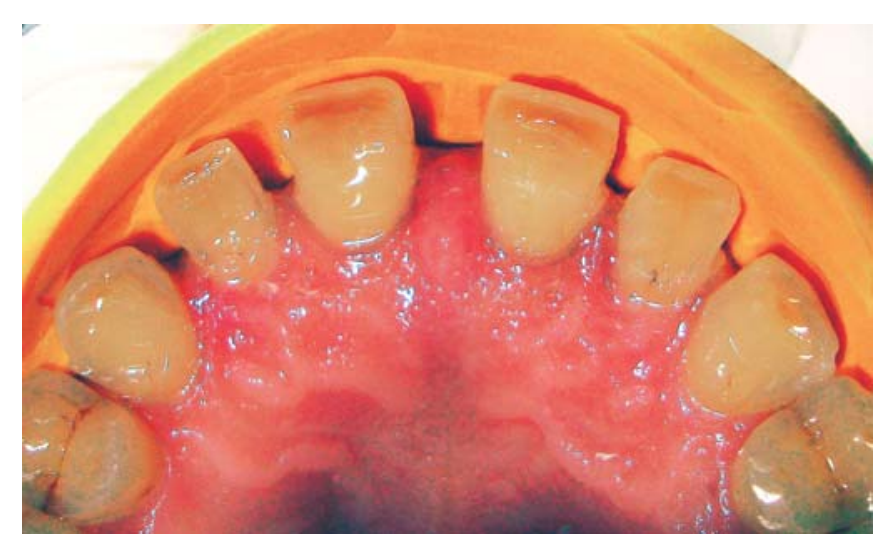

Fig. 5B: Tooth preparation guide 
was removed, washed, inspected, disinfected and sent to laboratory for fabrication of laminate veneers in lithium disilicate ceramics (IPS e.max, Ivoclar Vivadent, Amherst, USA). The laboratory was also sent the mock up, the pictures and the photographs of pre-evaluated temporaries. The technician was instructed about the shade, size and shape the patient had approved.

Another sets of provisional laminates were prepared using temporary resin materials in the silicone index (Protemp IV, 3M ESPE, USA). These were finished, polished and luted to the teeth with resin cement after spot etching.

\section{Luting of Laminates}

The provisional laminates were removed. The surfaces of all the prepared teeth were cleaned and pumiced to remove any remnants of the provisional luting agent. The laminate veneers were tried intraorally to see their fit, form, position, shape and shade. Once this was satisfactory, they were finally luted (Fig. 7).

The luting procedure was carried out initially over the central incisors, followed by the lateral incisors and finally the canines. The inner surface of the veneers was etched using 5\% hydrofluoric acid for 60 seconds and rinsed

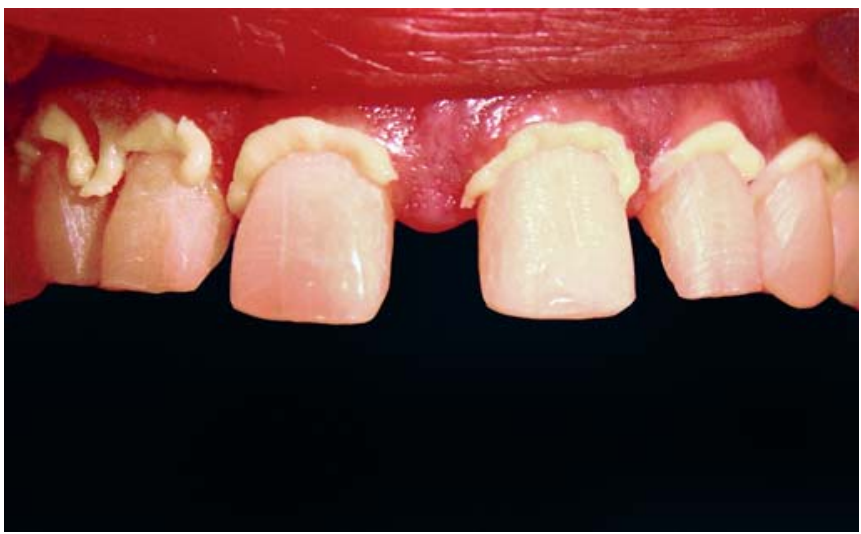

Fig. 6A: Gingival retraction

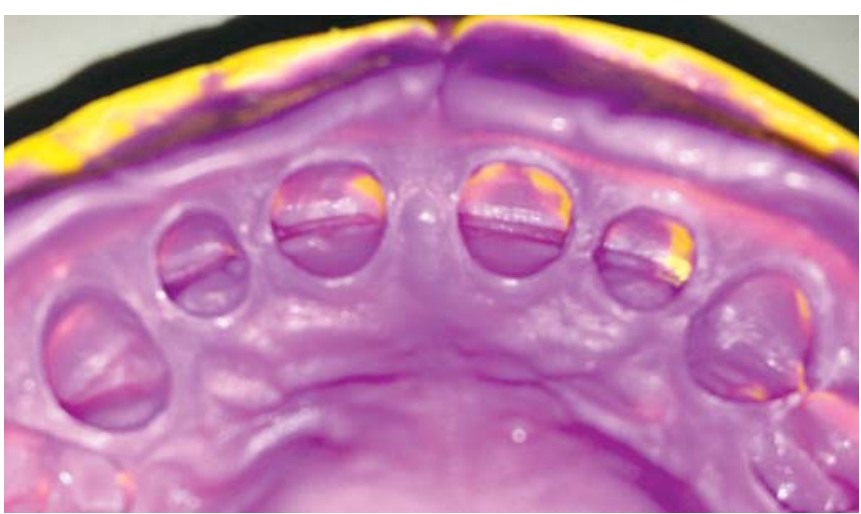

Fig. 6B: Final impression thoroughly. A plumber's tape was placed on adjacent teeth to protect them while the tooth surface was etched using 37\% Orthophosphoric acid (Etchant Gel, SS White, Germany) for 30 seconds and rinsed. Veneers were dried and Silane coupling agent (Silano, Angelus, Brazil) was applied over the etched surface. The tooth surface was coated with the bonding agent (Single Bond Universal, 3M ESPE, USA) and air dried. Resin cement (Variolink N, Ivoclar Vivadent, Amherst, USA) was coated over the veneers and was placed over the tooth surface. Care been taken to position the laminate from the cervical to the incisal edge for proper fitting.

A short light curing of 5 seconds was done to stabilize the position of the laminates and excess cement was removed to avoid the gingival irritation and fracture of the laminates. Interdental cement was removed using dental floss. Later, the final curing was carried out for 60 seconds. The same procedure was followed for luting of the lateral incisors and the canines. While etching and curing the adjacent teeth were always protected with a plumber's tape to prevent damage to the laminates. After the luting of all the six laminates, the patient was satisfied and happy with the outcome of the treatment (Figs 8 and 9). The patient was recalled for follow-up after a day, 1 week, 1 month and 6 months and has been functioning satisfactorily.

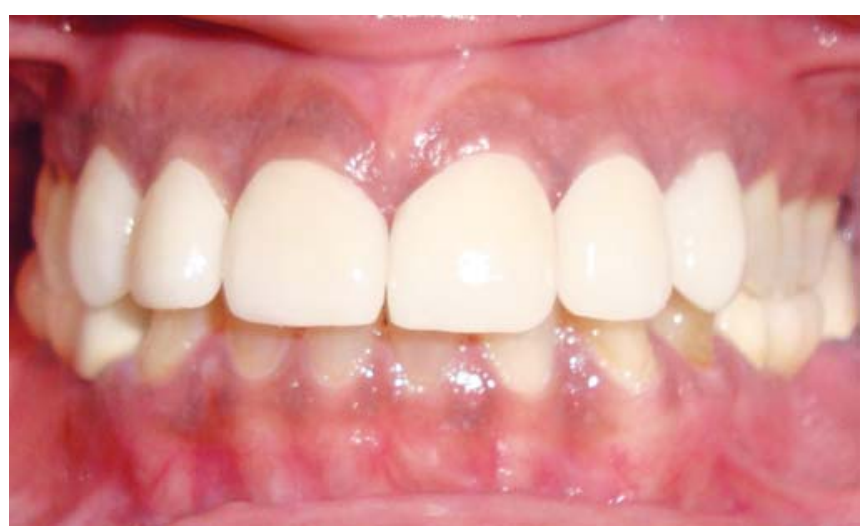

Fig. 7: Post-treatment intraoral view

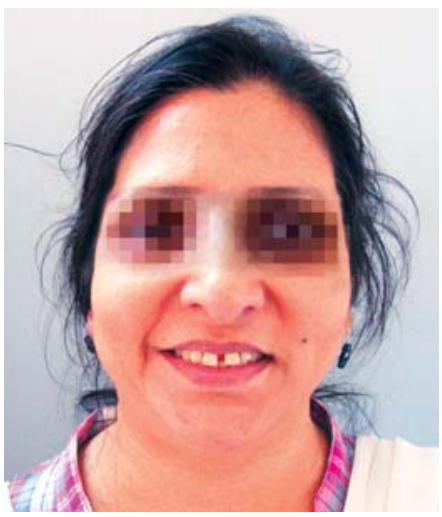

Fig. 8: Pretreatment photograph 


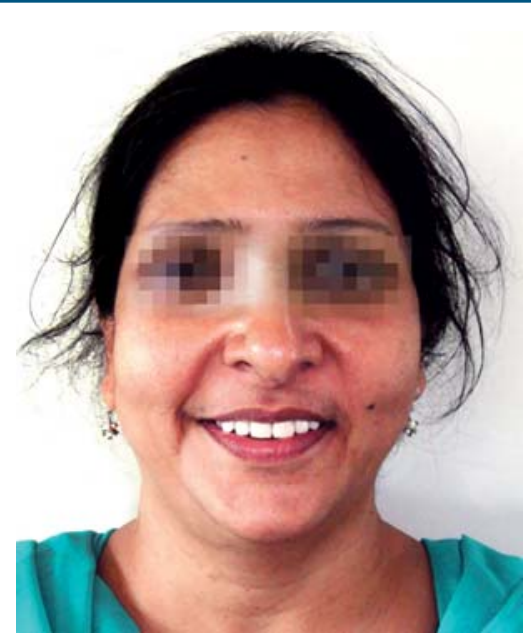

Fig. 9: Post-treatment photograph

\section{DISCUSSION}

Porcelain laminate veneers allowed successful restoration of function and esthetics with a minimally invasive procedure, resulting in a natural and pleasing smile. There are several options for treating anterior spaces, such as direct composite restorations, full veneer crowns, laminate veneers and, among these, the laminates are proved to be ideal restorative option as it requires a minimal invasive tooth preparation, resistance to discoloration unlike composites ${ }^{3}$ and has high degree of success rate.

Treating patients with porcelain laminate veneers need to have a blue print prior to carrying out the preparation. Preparing the pre-evaluated temporaries ${ }^{2}$ helped both the dentist and the patient to visualize and analyze the final outcome of the laminates. This could be done directly on the teeth intraorally or a diagnostic wax-up on the cast. In this case, the diagnostic wax-up was carried out and silicone index was made to prepare the pre-evaluated temporaries intraorally. This saved the chair-side time. Minimum preparation of the tooth also helped to preserve the enamel to get a more effective bond between the laminates and the tooth surface when luted with the resin cements. ${ }^{2}$ In this case, a butt type of preparation for the incisal edge was carried out as a study in 2009 by Ghani Mirra and Salem Mahalawy ${ }^{4}$ found out that incisal butt type of preparation had the highest mean values of fracture strength as compared to feather edge and incisal overlap type of preparation. Also, they found out that the marginal microleakage was higher in incisal overlap type of preparation.

The veneers were fabricated in lithium disilicate ceramics knowing its high degree of translucency and strength $^{5}$ as compared to conventionally used low fusing feldspathic porcelains. The veneers were luted using resin cement Variolink N bonding system. Thineers or ultrathin veneers ${ }^{6}$ have also been a choice for minimum preparation laminates. However, in this case, thineers were not indicated because of the wider diastema.

\section{CONCLUSION}

Porcelain laminate veneers are a conservative approach. With the latest in Dentistry, believing in the concept of minimal invasive procedures for preservation of tooth structure, porcelain laminate veneers would be the ideal choice for restoring spacing between the anterior teeth. If properly indicated and executed, these restorations have a long-term success rate. Following a strict protocol of evaluation, pre-evaluative temporization, tooth preparation, properly selected material for fabrication of laminates and meticulous luting will enhance the outcome and the longevity of the treatment.

\section{REFERENCES}

1. Trevor Burke FJ. Survival rates for porcelain laminate veneers with special reference to the effect of preparation in Dentin: A literature review. J Esthet Restor Dent 2012:24;257-265.

2. Gurel G, Morimoto S, Calamita M, Coachman C, Sesma N. Clinical performance of porcelain laminate veneers: outcomes of the esthetic pre-evaluation temporary (APT) technique. Int J Periodontics Restorative Dent 2012;32(6):625-635.

3. Meijering AC, Roeters FJM, Mulder J, Creugers NHJ. Patient's satisfaction with different types of veneer restoration. J Dent 1997;25(6):493-497.

4. GhaniMirra AE, Mahalawy SE. Fracture strength and microleakage of laminate veneers. Cairo Dental Journal 2009;25(2):245-254.

5. Drummond JL, King TJ, Bapna MS, Koperski RD. Mechanical property evaluation of pressable restorative ceramics. Dent Mater 2000;16:226-233.

6. Kacker MD, Yarovesky U, Jadali L. Ultra-thin veneers: beautiful and natural. Dent Today 2011;30(7):102,104-105.

\section{ABOUT THE AUTHORS}

\section{Prasad Nanasaheb Mhaske (Corresponding Author)}

Postgraduate Student, Department of Prosthodontics, MGM Dental College and Hospital, Navi Mumbai, Maharashtra, India, Phone: 09022776222,e-mail:prs_003@hotmail.com

\section{Sabita M Ram}

Professor and Head, Department of Prosthodontics, MGM Dental College and Hospital, Navi Mumbai, Maharashtra, India

\section{Naisargi Shah}

Professor, Department of Prosthodontics, MGM Dental College and Hospital, Navi Mumbai, Maharashtra, India

\section{Jyoti B Nadgere}

Professor, Department of Prosthodontics, MGM Dental College and Hospital, Navi Mumbai, Maharashtra, India 